\title{
PNP Gene
}

National Cancer Institute

\section{Source}

National Cancer Institute. PNP Gene. NCI Thesaurus. Code C43239.

This gene is involved in purine catabolism. 